\title{
Lesson from the COVID-19 pandemic: pathologists need to build their confidence on working in a digital microscopy environment
}

\author{
Rodolfo Montironi $^{1}$ (i) $\cdot$ Alessia Cimadamore ${ }^{1} \cdot$ Liang Cheng $^{2} \cdot$ Antonio Lopez-Beltran $^{3} \cdot$ Marina Scarpelli $^{1}$
}

Received: 21 January 2021 / Revised: 6 April 2021 / Accepted: 14 May 2021 / Published online: 25 May 2021

(c) The Author(s), under exclusive licence to Springer-Verlag GmbH Germany, part of Springer Nature 2021

We read with interest the paper published by RodriguesFernandes and colleagues in the October issue of Virchows Archiv, entitled "The use of digital microscopy as a teaching method for human pathology: a systematic review" [1]. As reported by the authors, "digital microscopy (DM) has become a useful alternative to conventional light microscopy (CLM)" [1].

Due to the constrains in this perduring time of pandemic, pathologists are reconsidering the traditional approach in examining glass slides with CLM and are becoming enthusiastic about the application of DM. The constrains are related to issues pathologists are facing, including the need of keeping physical distance with colleagues and laboratory professionals, thus hampering social interaction and reducing their visibility. Such constrains can have a negative impact on their relationship with clinicians, patients, and students [2].

The question is what pathology will be like after the resolution of the pandemic, while remaining an integral part in the clinical processes. Considering that medical practices, including pathology, are moving into an era of global digitalization, the pathology practice might not return to the CLM routine followed before the pandemic [2].

Rodolfo Montironi and Alessia Cimadamore contributed equally to this work.

Rodolfo Montironi

r.montironi@univpm.it

1 Section of Pathological Anatomy, Polytechnic University of the Marche Region, School of Medicine, United Hospitals, Via Conca 71, 60126 Ancona, Italy

2 Department of Pathology and Laboratory Medicine, Indiana University School of Medicine, Indianapolis, IN, USA

3 Department of Morphological Sciences, Cordoba University Medical School, Cordoba, Spain

\section{From a projection screen to a virtual slide}

The current approach adopted to examine glass slides represents the technological and cultural evolution pathologists have been involved with since the 1970s. The successive phases in such evolution have been summarized in a recent publication [3], starting from the traditional optical microscopy, i.e., lonely view of a glass slide by an individual pathologist, and microscope image shown on projection screen or on a television via an analog camera to digital imaging and computer monitor and virtual slide. Pallua et al. have commented on such changes, all leading toward a greater role of digitalization in pathology [3].

\section{The rocky road to $D M$}

DM was born approximately 40 years ago [4]. Professor Peter H. Bartels (University of Arizona) contributed to the development of this field. Together with others, he developed the theoretic background of image analysis as well as several applications. The recording and merging process were extended over a large area, using multi-megapixel arrays. The viewer could roam throughout the large arrays for an evaluation of the tissue features. It corresponds to what we call virtual slide. This included machine vision and case-based reasoning, i.e., the current bases of the artificial intelligence [5].

Bartels' fields of interest also included digitalization in uropathology. One of his works focused on the cribriformity index of prostate cancer. At that time, this could have appeared of scarce clinical significance. Nowadays, prostate cancer with cribriform architecture is the most aggressive form of Gleason pattern 4 [3]. Two authors of this current contribution (RM and MS) had the opportunity of collaborating with him in the early 1990s and to witness the development of a miniaturized microscope array digital slide scanner [5]. 
A recent paper by Paul J. van Diest and colleagues entitled "Rocky road to digital diagnostics: implementation issues and exhilarating experiences" described and retraced the path they followed since 2007 in the implementation of DM in their laboratory [6].

\section{Working in a digital microscopy environment}

Pathologists need to acquire a high level of confidence when evaluating virtual slides on a computer monitor, changing magnification, and roaming throughout the image as with CLM.

A training program has been developed in Ancona, Italy, to acquire such "easiness." This has included an optical microscope with digital camera and TV monitor. Such a system allows the pathologist to examine the section under the microscope and then the same image on the monitor and vice versa. The aim is to get the pathologist acquainted with what he/she is used to seeing with CLM and then what he/she can see on the monitor and match the two sources of images. The microscope is usually used at low magnification to have the whole tissue section or most of it on the monitor. Magnification and field of view are changed as needed. The training starts with normal tissue. This is followed by the examination of simple lesions, such as basal cell carcinoma of the skin (see the didactic schematic drawing, Fig. 1). This is then followed by the examination of more complex lesions, such as prostate biopsies and whole mount sections of radical prostatectomies, i.e., one of our major interest.

The whole process requires about a week of work. After completing it, pathologists feel confident with moving from glass slide to virtual slides examination. Confident means that the pathologists feel at ease with seeing images and not fields of view in the microscope and, at the same time, change magnification and moving around the image. This is a crucial phase when planning to shift from glass slide to
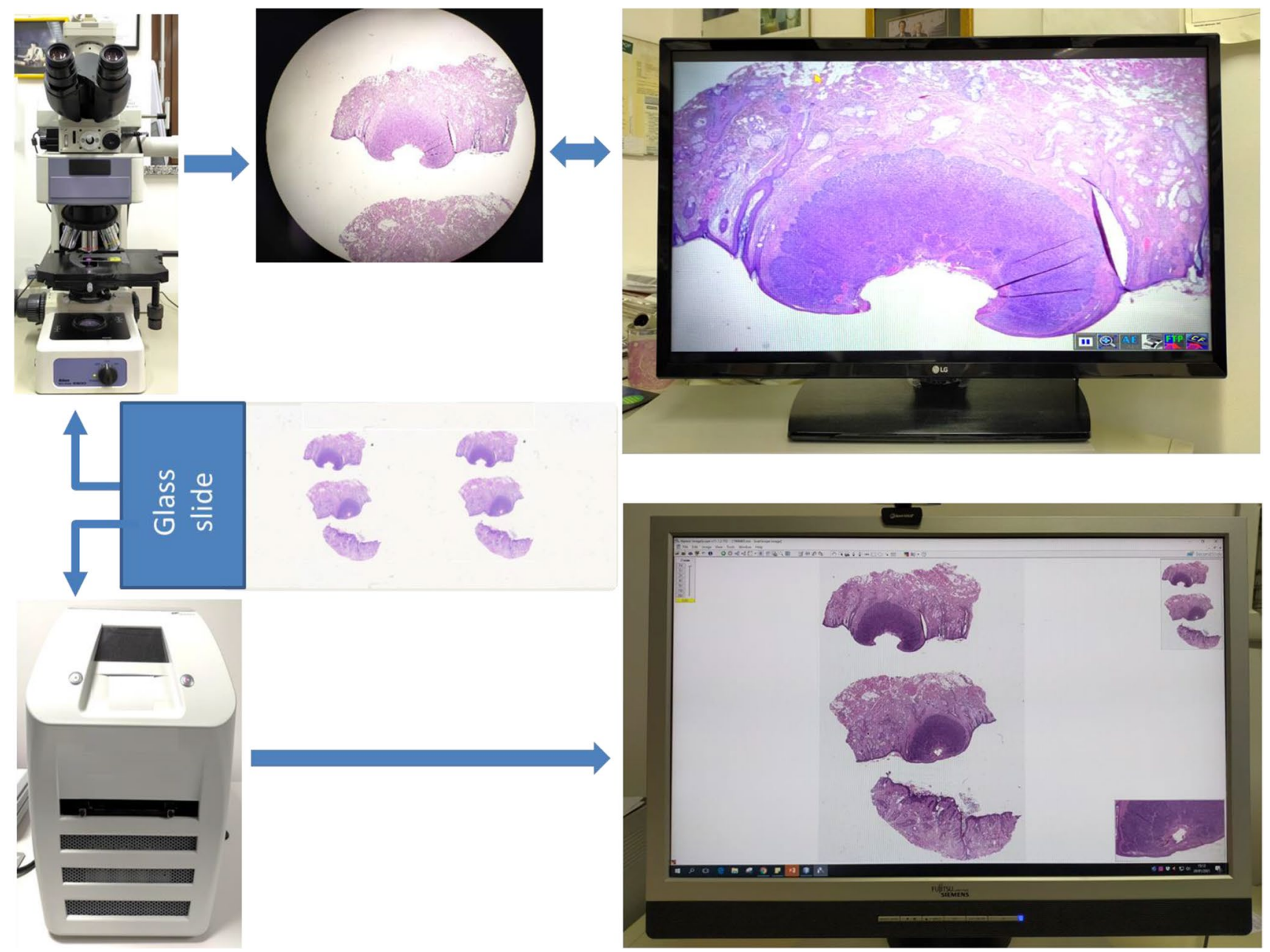

Fig. 1 The didactic schematic drawing. From glass slide to conventional light microscope and an image shown on a TV monitor to digital scanner and a virtual slide seen on a computer monitor 
virtual slide. Pathologists, not undergoing such intermediate phase, can take much longer to get used to dealing with virtual cases.

Retamero et al. [7] have dealt with an issue similar to ours. With regard to time needed to transition from CLM to DM, $26 \%$ of pathologists reported being ready in less than $24 \mathrm{~h}, 61 \%$ needed between 1 and 7 days, and the remaining $13 \%$ between 7 and 14 days.

\section{What the situation could be like in $\mathbf{5}$ years from now}

For sure, routine digital diagnostics in pathology will become routine in many centers, aiming at replacing CLM with DM. However, we have to think that digital diagnostics in pathology depends on the routine work done by a new types of laboratory professionals as well as availability of faster scanners and increased storage capability [6]. There might be a further role for fluorescence confocal microscopy. The acquisition of digital images with this approach required 2 to $5 \mathrm{~min}$, without the need for conventional processing nor equipped laboratory or dedicated personnel, with interpretation done remotely and even in smart working [8]. With multiplex staining on a single slide and high-resolution image analysis, machine vision and deep learning techniques could offer diagnostic algorithms that can be adopted for precise diagnosis, prognosis, and prediction to response to therapy of prostate cancer patients [9]. The adoption of digital pathology will enable the implementation of artificial intelligence (AI)-based algorithms in the routine practice. These algorithms have the potential to go beyond the visual assessment of the main histopathological features to capture subtle patterns which are currently beyond human recognition [10].

The question is whether DM will replace permanently CLM. It is difficult to foresee a distant future. However, the way we do pathology is related to CLM and this has to remain at the basis of our profession, at last for the next few years. That is why we need to establish a double LCM/DM workflow as already done in Utrecht [6].

We focused here on prostate cancer. This is due to the fact that prostate cancer is one of the main topic of research and routine work of the authors of this contribution. Even though we have limited expiring on other tumors of organs other than those included in the uropathology field, we are fully aware of the fact that what was reported here in relation to prostate cancer, others, including Stathonikos et al. [6] have had a similar experience on other organs and types of pathologies. In their recent contribution, Stathonikos et al. [6] also summarized the potential pros and cons of digital pathology.
Authors' contributions RM, AC: drafting of the manuscript; MS: conception of the manuscript; LC, ALB: literature review.

\section{Declarations}

This is only partially applicable since the manuscript is an editorial and does not provide research data. Research involving human participants and/or animals is not reported. There was no need for an informed consent.

Conflict of interest The authors declare no competing interests.

\section{References}

1. Rodrigues-Fernandes CI, Speight PM, Khurram SA, Araújo ALD, Perez DEDC, Fonseca FP, Lopes MA, de Almeida OP, Vargas PA, Santos-Silva AR (2020) The use of digital microscopy as a teaching method for human pathology: a systematic review. Virchows Arch 477(4):475-486

2. Montironi R, Cheng L, Cimadamore A, Lopez-Beltran A, Scarpelli M (2020) Uropathologists during the COVID-19 pandemic: what can be learned in terms of social interaction, visibility, and social distance. Eur Urol 78(3):478-481

3. Montironi R, Cimadamore A, Scarpelli M, Cheng L, LopezBeltran A, Mikuz G (2020) Pathology without microscope: from a projection screen to a virtual slide. Pathol Res Pract 216(11):153196. https://doi.org/10.1016/j.prp.2020.153196

4. Cimadamore A, Lopez-Beltran A, Scarpelli M, Cheng L, Montironi R (2020) Digital pathology and COVID-19 and future crises: pathologists can safely diagnose cases from home using a consumer monitor and a mini PC. J Clin Pathol 73(11):695-696

5. Montironi R, Cimadamore A, Lopez-Beltran A, Cheng L, Scarpelli M (2021) Exciting experiences in the 'Rocky road to digital diagnostics.' J Clin Pathol 74(1):5-6

6. Stathonikos N, Nguyen TQ, van Diest PJ (2020) Rocky road to digital diagnostics: implementation issues and exhilarating experiences. J Clin Pathol :jclinpath-2020-206715. https://doi.org/10. 1136/jclinpath-2020-206715

7. Retamero JA, Aneiros-Fernandez J, Del Moral RG (2020) Microscope? No, Thanks: user experience with complete digital pathology for routine diagnosis. Arch Pathol Lab Med 144(6):672-673

8. Rocco B, Sighinolfi MC, Sandri M, Spandri V, Cimadamore A, Volavsek M, Mazzucchelli R, Lopez-Beltran A, Eissa A, Bertoni L, Azzoni P, Reggiani Bonetti L, Maiorana A, Puliatti S, Micali S, Paterlini M, Iseppi A, Rocco F, Pellacani G, Chester J, Bianchi G, Montironi R (2020) Digital biopsy with fluorescence confocal microscope for effective real-time diagnosis of prostate cancer: a prospective, comparative study. Eur Urol Oncol :S2588-9311(20)30136-X. https://doi.org/10.1016/j.euo. 2020.08.009

9. Huss R, Coupland SE (2020) Software-assisted decision support in digital histopathology. J Pathol 250(5):685-692

10. Rakha EA, Toss M, Shiino S, Gamble P, Jaroensri R, Mermel CH, Chen PC (2020) Current and future applications of artificial intelligence in pathology: a clinical perspective. J Clin Pathol :jclinpath-2020-206908. https://doi.org/10.1136/jclinpath-2020-206908

Publisher's note Springer Nature remains neutral with regard to jurisdictional claims in published maps and institutional affiliations. 